\title{
Synthetic Jet Flow Field Database for CFD Validation
}

\author{
Chungsheng Yao*, Fang Jenq Chen ${ }^{\dagger}$, Dan Neuhart ${ }^{\ddagger}$, and Jerome Harris ${ }^{\S}$ \\ NASA Langley Research Center, Hampton, VA 23681
}

\begin{abstract}
An oscillatory zero net mass flow jet was generated by a cavity-pumping device, namely a synthetic jet actuator. This basic oscillating jet flow field was selected as the first of the three test cases for the Langley workshop on CFD Validation of Synthetic Jets and Turbulent Separation Control. The purpose of this workshop was to assess the current CFD capabilities to predict unsteady flow fields of synthetic jets and separation control. This paper describes the characteristics and flow field database of a synthetic jet in a quiescent fluid. In this experiment, Particle Image Velocimetry (PIV), Laser Doppler Velocimetry (LDV), and hot-wire anemometry were used to measure the jet velocity field. In addition, the actuator operating parameters including diaphragm displacement, internal cavity pressure, and internal cavity temperature were also documented to provide boundary conditions for CFD modeling.
\end{abstract}

\section{Nomenclature}

$\begin{array}{ll}d & =\text { diaphragm displacement } \\ h & =\text { jet exit slot width } \\ f & =\text { forcing frequency } \\ u & =\text { velocity component along the slot } \\ v & =\text { velocity component across the slot } \\ w & =\text { jet streamwise component } \\ \boldsymbol{x} & =\text { along-slot coordinate } \\ y & =\text { across-slot coordinate } \\ z & =\text { jet streamwise coordinate } \\ p & =\text { cavity pressure } \\ T & =\text { cavity temperature } \\ R_{\text {max }} & =\text { Reynolds based on maximum jet velocity and slot width }\end{array}$

\section{Introduction}

\begin{abstract}
Three test cases were proposed for the Langley Research Center Workshop of CFD Validation of Synthetic Jets 1 and Turbulent Separation Control ${ }^{1}$. The flow field of an isolated oscillatory jet with zero net mass flow was selected as the first test case. This basic flow field is generated by a stand-alone synthetic jet actuator in quiescent air. The actuator has a rectangular slot to produce a two-dimensional plane synthetic jet at near field, and provide a case for 2D CFD validation. In the far field, the roles of three dimensionality and turbulence will become increasingly significant. The second and third cases of this workshop raise the flow complexity for the CFD challenge. The second case was a round oscillatory jet in a crossflow with zero streamwise pressure gradient. This complex flow, highlights the flow interaction and three dimensionality of a developing jet within a turbulent boundary layer. The third case selected involved the control of separated flow over a wall mounted hump model by means of both steady suction and oscillatory jets.
\end{abstract}

\footnotetext{
- Aerospace Engineer, Flow Physics and Control Branch, MS 170 Hampton, VA 23681.

† Aerospace Engineer, Flow Physics and Control Branch, MS 170 Hampton, VA 23681.

‡ Aerospace Engineer, Flow Physics and Control Branch, MS 170 Hampton, VA 23681.

$\S$ Optics Technician, Flow Physics and Control Branch, MS 170 Hampton, VA 23681.

Copyright $(2004$ by the American Institute of Aeronautics and Astronautics. The U.S. Government has a royalty-free license to exercise all rights under the copyright claimed herein for Government purposes. All other rights are reserved by the copyright owner.
} 
Among the three test cases selected, the basic oscillating jet flow field is the least complicated, both for the flow itself and the required flow field database. The main characteristic is flow unsteadiness with additional properties of low Reynolds number $\left(\operatorname{Re}_{\max } \sim 3500\right.$ ), vortex pair motion, and transition to turbulence.

In this study, hot-wire anemometry, Laser Doppler Velocimetry (LDV) and Particle Image Velocimetry (PIV) were used to acquire the synthetic jet flow field over a complete drive cycle. Each measurement technique has its own advantages and limitations. Independent measurements from three different techniques offer a cross check of the flow field data. Specifically, the oscillatory jet flow fields in a 2D plane along and across the slot were measured by PIV. The LDV and hot-wire probe were set up to scan the jet velocity profile along the centerline of the jet. The actuator operating parameters including diaphragm displacement, internal cavity pressure, and internal cavity temperature were monitored to provide boundary conditions for CFD simulations.

Data supplied to the workshop (available on the website) is different than the data discussed in this paper. The actuators studied in this report were installed with a new diaphragm, which produced a higher and more stable jet output. However, the overall behavior of the flow field is similar.

In recent years synthetic jets have demonstrated promising flow control applications ${ }^{2}$ among active flow control devices $^{3-5}$ developed. Previous studies have covered the development of the actuator as well as the general behavior and performance of the synthetic jet produced. The actuator may be driven by either an acoustic loudspeaker, or a piston, or a piezoceramic diaphragm. Previous flow measurements were less geared toward the collection of a detailed flow field database over an extended driving phases, with little or no attention given to input boundary conditions. Smith and Glezer(1998) ${ }^{6}$ measured the velocity profile of plane jets from rectangular slot with hot-wire and flow visualization. Velocity profiles covered a height range of 40 slot widths $(h)$ with the jet actuator operating at $1.14 \mathrm{KHz}$. The induced jet velocity from the rising vortex pair reached its peak near $4 h$ above the slot while, also the suction motion was limited to near $4 \mathrm{~h}$ above the jet exit. Crook, et al. (1999) ${ }^{7}$ studied the development of a round jet using an analytical model and compared his results with hot-wire measurements. The study was focused on the maximum velocity from the jet as function of actuator geometry and operation parameters. Peak frequency was about $1.4 \mathrm{KHz}$. Mallinson, et al. (1999) ${ }^{8}$ studied a round jet near $1.45 \mathrm{KHz}$ with variable orifice diameters and cavity heights using a single component hot-wire. Chen, et al. $(2000)^{9}$ provided velocity profiles at the centerline of a plane jet, and jet exit velocity profiles along and across the jet slot with a single component hot-wire. Chen used an actuator with a diaphragm oscillating at a frequency of 500 and $1000 \mathrm{~Hz}$. Muller, et al $(2001)^{10}$ used PIV to measure the detailed flow field of a circular jet driven by a speaker vibrating between $20 \sim 200 \mathrm{~Hz}$. Their main focus was on thrust from the synthetic jet and its flow structure. PIV data were taken only at a 30 degree phase interval with a PIV measurement resolution about $1 / 3$ of the orifice. In this case, the uncertainty of velocity measurements due to the velocity gradient within the measurement volume increases significantly, specifically for maximum jet speed. Bera, et al (2001) ${ }^{11}$ used PIV to map plane synthetic jet flows from a 2D slot. Both purely synthetic jet and a mixed jet containing an additional blowing were investigated. An acoustic speaker drove the jets at $200 \mathrm{~Hz}$. Only eighteen phases of PIV data were collected at a PIV spatial resolution of $1 \mathrm{~mm}$, which is on the same order of the slot width. Therefore, the velocity profiles near the exit were not properly resolved. Guy, et al $\left(2001^{12}, 2002^{13}\right)$ studied a plane synthetic jet produced by piezo-diaphragm with hot-wire measurements. Similar to Chen $(2000)^{9}$, they observed two resonance frequencies, in this case, 700 and $1160 \mathrm{~Hz}$. Maximum jet velocity was measured at $\sim 12 h$ instead of $\sim$ $4 h$ from Smith and Glezer.

The objective of this experiment was to establish a detailed flow field database from a synthetic actuator operating under a selected condition. It was not intended to be a parametric survey or performance optimization of synthetic jets. Issues involved with this test, and some prominent flow features observed are presented.

\section{Experiment Setup}

\section{A. Synthetic jet actuator}

The synthetic jet actuator for this experiment was based on an earlier design studied in detail by Chen et al $(2000)^{9}$. The pumping cavity chamber is in-line with the jet slot for compact design (Figure 1). The jet exit slot of the actuator is $1.25 \mathrm{~mm}$ wide and $35.56 \mathrm{~mm}$ long. The slot width was enlarged compared to the previous design to allow velocity profiles across the slot to be properly resolved for PIV and LDV measurements. The actuator is flush mounted on an aluminum floor plate, and is covered by a glass $(0.25$ inch thick) enclosure, $2 \mathrm{ft} \times 2 \mathrm{ft} \times 2 \mathrm{ft}$ in dimension. The glass enclosure isolates the synthetic jet flow from the ambient air and also serves to contain the seeding particles for LV and PIV measurements. A sliding door on the glass panel provides access for injecting seed and the hot-wire probe. The jet is located at the center of the floor, and the slot is parallel to the glass sidewall. 


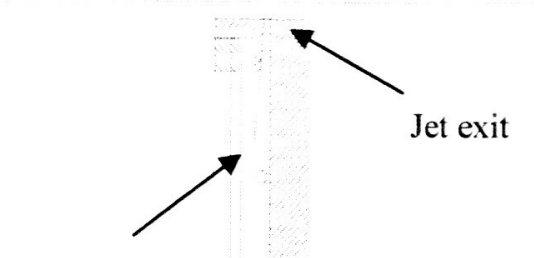

Diaphragm

Figure 1. Schematic diagram of cavity

The actuator has a Plexiglas housing with a narrow cavity beneath the slot. The jet flow was driven by a single piezoelectric diaphragm, 2 inches in diameter, mounted on one side of a narrow cavity. The piezo diaphragm was driven at $445 \mathrm{~Hz}$, which was selected to operate slightly away from the actuator resonant frequency near $450 \mathrm{~Hz}$. Figure 2 shows the velocity output of the actuator as a function of the forcing frequency. An O-ring seal, 1.85 inch in diameter, is clamped between the diaphragm and the actuator cavity. The maximum jet velocity generated could reach $30 \sim 50 \mathrm{~m} / \mathrm{s}$, depending on the driver voltage, actuator performance, and the aging of actuator. In a routine test, there was no performance degrading but a slight phase variation with test time, which normally lasts between 5 to 10 hours. The actuator did indicate a gradual degrading of the pressure level over a long period of months of usage. In these cases, diaphragm displacement was a preferred solution. The diaphragm may need to be replaced due to cabling faults or the build-up of seeding material inside the cavity.

\section{B. Actuator parameters}

Three actuator operating parameters including diaphragm displacement $(d)$, cavity pressure $(p)$ and cavity temperature $(T)$, were measured to monitor the actuator performance, and to provide boundary conditions for computation and actuator modeling (Figure 3). A fiber optics displacement sensor was installed to measure the diaphragm displacement at the center of the piezoceramic disc. The fiber optic probe was calibrated in-situ using a micrometer. The actuator diaphragm displacements oscillated between about $0.3 \mathrm{~mm}$ inward and $0.5 \mathrm{~mm}$ outward. The total amplitude within $0.8 \mathrm{~mm}$ is small compared to the static cavity depth of $4.8 \mathrm{~mm}$, including the $\mathrm{O}$ ring spacer. A dynamic pressure transducer installed at the center of the sidewall of the actuator (the fixed wall) opposite of the diaphragm monitors the cavity pressure waveforms. A thermocouple device was installed at the bottom of the cavity to monitor the internal temperature. The flow geometry and coordinates are shown in Figure 4.

The experiment was repeated separately for the three measurement techniques. Different diaphragms were installed in the actuator for each test due to actuator failures or changes found in the actuator performance. Hot-wire tests were conducted in clean air while the glass enclosure was moderately seeded for LDV measurements, and heavily seeded for PIV measurements. No changes of the pressure waveforms were detected under different seeding conditions.

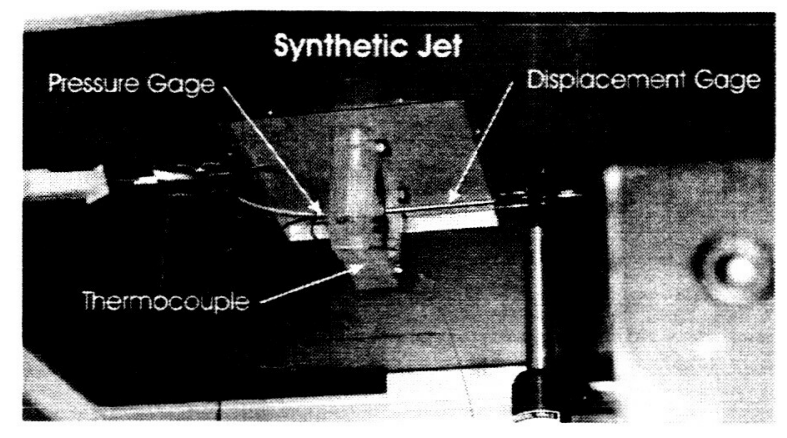

Figure 3. Synthetic actuator and sensors for pressure, displacement, and temperature

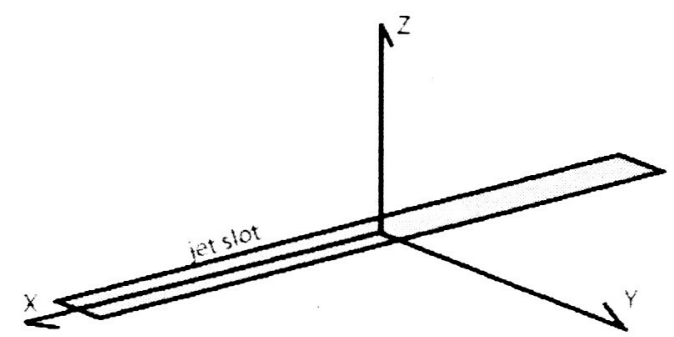

Figure 4. Synthetic jet coordinates. 


\section{Flow Field Measurement}

\section{A. Hot-wire Measurements}

Hot-wire Anemometry provides a measure of the velocity at a single point for the velocity component perpendicular to the sensing wire. In the current setup, hot-wire measurements were made with a constanttemperature anemometer (CTA) and a single-sensor hot-wire probe. The sensing element of the hot-wire probe, 5 $\mu \mathrm{m}$ in diameter and $1.25 \mathrm{~mm}$ long, is a platinum-plated tungsten wire operated at an overheat ratio of 1.8 .

The probe was mounted perpendicular to the floor with the sensing element located at the center of the slot and parallel to the long axis of the slot. Measurements were taken at 47 stations with height ranging to $50 \mathrm{~mm}$ above the slot. A hot-wire probe support was mounted on a translation stage with a computer-controlled traversing system installed on the exterior of the glass enclosure. A slotted opening on the sidewall provides the access into the glass enclosure.

The hot-wire probe was calibrated with a commercial desktop calibration unit. The signals from the CTA outputs (non-linearized) were sampled at $100 \mathrm{kHz}$ with 16,384 data points recorded at each station. At a forcing frequency of $445 \mathrm{~Hz}$, there were 72 periods of an input waveform recorded. Within each period of the driving cycle there were 225 data points. Seventy-two samples were averaged to compute the phase-locked statistics. Hot-wire signals were de-rectified to obtain negative velocities during the actuator suction cycle at lower stations. Uncertainty was $+/-2.5$ $\%$ for measurements at heights of $5 \mathrm{~mm}$ or higher and $+/-6 \%$ for stations near the solid surface.

\section{B. Laser Doppler Velocimetry Measurements}

Similar to hot-wire measurements, LDV is single point in space measurement technique. LDV signals provide a velocity record and the corresponding particle arrival time whenever a valid signal is detected. In this test, a LDV system was set up to measure the vertical jet velocity and its decay along the centerline of the slot. Vertical velocity measurements were made at 47 locations above the rectangular slot between 0 to $70 \mathrm{~mm}$. The transmitted laser beams from a fiber optic probe were projected through the glass sidewall in a direction parallel to the long axis of the rectangular jet slot, and in a vertical plane. The crossing point of the two laser beams, which is the LDV sample volume, was centered over the center of the slot, both longitudinally and laterally. The scattered light from the seeding particles was collected by a second fiber optic probe, which was located in the forward-scatter direction, 50 degrees off of the direct forward scatter axis.

The seeding particles were $0.9 \mu \mathrm{m}$ polystyrene latex spheres (PSL, with specific gravity at 1.04), suspended in 200-proof ethanol. Based on a first-order estimation, particles $\sim O(1 \mu \mathrm{m})$ follow the flow well at the applied frequency. The argon-ion LV system utilized the $514.5 \mathrm{~nm}$ (green line). The cross-beam half angle between the two transmitted beams was 1.872 degrees, calibrated at the far field $(\sim 25 \mathrm{ft})$ via a beam separation measurement. The LDV optics had a $750.5 \mathrm{~mm}$ focal length. The estimated sample volume cross-section was about 175 microns. The transmitting probe was set at an angle of 4 degrees down relative to the horizontal plane to accommodate getting the sample volume into the slot at $0 \mathrm{~mm}$ height without blocking the lower beam. The receiving probe was set at an angle of 10 degrees down to ensure that all available scattered light was collected unobstructed. The probes were mounted on translation stages to provide lateral and vertical motion. The stages have a position accuracy of 1.0 micron. Once alignment was achieved between the transmission and receiving probes, each was moved individually to the new position.

LDV signals from the scattering particles were processed using FFT processors. Doppler frequency, hence velocity signal, was estimated based on 256 point FFT spectrum analysis at each particle arrival. The drive signal waveform was also recorded along with particle arrival time to determine the phase information of the velocity signal.

At each station, 30,000 data points were collected in a period of several minutes, depending on the seeding density. Based on the phase information collected at each data point, the 30,000 data points were sorted into 36 phase bins, at 10 degree of phase interval. Within each bin, the number of samples may vary from a few hundred to over 2000. Theses samples were then used to compute phase-locked statistics.

The uncertainty in the data was calculated using estimates of bias and precision errors in the experiment. The estimates were based on a nominal condition using an approximate mean velocity in the jet. The bias estimates were based on experimental geometrical parameters, LDV processor bias, and biases related to the seeding material used. The total bias and the precision were propagated through the coordinate transformation due to the probe downward tilt and combined to give an estimate of the total uncertainty in the vertical velocity measured of $+/-0.54 \mathrm{~m} / \mathrm{sec}$. Mean velocities calculated using 15,000 samples were within $1.8 \%$ of those calculated based on 30,000 samples. 


\section{Particle Image Velocimetry Measurements}

PIV measures an instantaneous velocity field over a grid of points in a plane in the fluid. In this test, a Digital PIV system was set up to measure the horizontal and vertical velocity components synchronized with the $445 \mathrm{~Hz}$ drive signal. The current PIV system includes $1024 \times 1280 \mathrm{CCD}$ cameras installed with a $200 \mathrm{~mm}$ Macro lens for the near field and a $105 \mathrm{~mm}$ Macro lens for large flow field measurements. The camera lens was placed approximately 12 inches from the laser sheet to cover a field of view between 9 to $30 \mathrm{~mm}$ wide. The magnification of the imaging optics was calibrated with an optical grid target aligned with the laser sheet. The accuracy of calibration was within $+/-1$ pixel over 1240 pixels. Interrogation resolution was set between 28 to 32 pixels corresponding to about 0.22 $\mathrm{mm}$ of measurement area for near field data and $0.77 \mathrm{~mm}$ for the large field of view test.

Dual Nd-Yag lasers, operated at $5 \mathrm{~Hz}$ rate and $100 \mathrm{~mJ}$ output per pulse, were used to illuminate a light sheet between $0.3 \sim 0.5 \mathrm{~mm}$ thick. The laser light sheet was projected perpendicular to the slot at the mid-section for $y-z$ plane measurements, and parallel to the slot for $x-z$ plane measurements. Laser pulse separations ( $\delta t$ between double exposure) were adjusted between $1.1 \sim 8.0 \mu$ s at each phase to maximize PIV performance ( 8 to 10 pixels of maximum particle displacement). In general, PIV interrogation software resolves between 1/10 to 1/20 sub-pixel resolution. The system has been shown to measure within $1 \%$ of free stream velocity in wind tunnels.

Both smoke and polystyrene latex (PSL) spheres were used to seed the glass enclosure. PIV measurements normally require higher seeding density than LDV to ensure its performance, i.e., high validation rate over the entire grids. Smoke particles were produced by a smoke generator using standard smoke fluids (specific gravity at 1.022). Smoke particles have a poly-disperse size distribution, which may cover from sub-micron to tens of micron while PSL particles are mono-disperse in size when manufactured. Some large agglomerated PSL particles may still be present in the flows when sprayed into the test chamber with water-ethanol mixture. Estimates based on the simple drag law indicate that particles less than $3 \mu \mathrm{m}$ should follow the oscillatory flow well while particles larger than 5 $\mu \mathrm{m}$ may introduce flow-tracking error. There was no particle sizer available at the test for in-situ particle size distribution measurements.

Both seeding particles, smoke and PSL, were tested to determine tracking errors based on the PIV measurements. There was only slight difference in a few of the velocity profiles measured at jet positive phases as

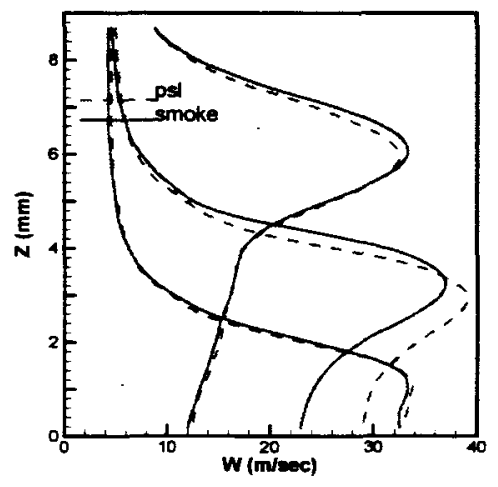

Figure 5. Comparison of jet profiles measured by smoke and PSL seeding

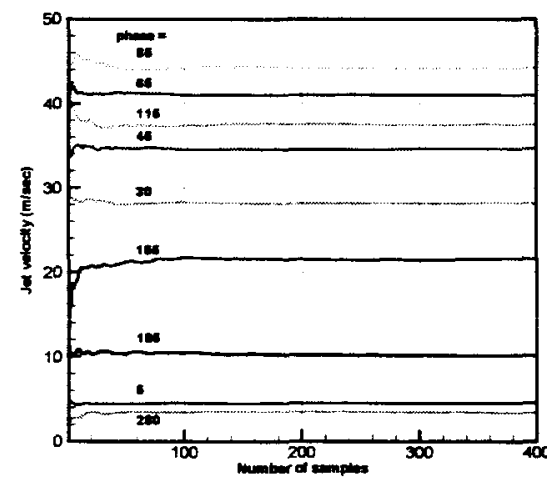

Figure 6. Convergence of mean velocity estimation.

shown for the three phases in (Figure 5). In the large field of view case, PSL seeding provides satisfactory seeding density for PIV signals. At high magnification PIV measurement for near field case, smoke particles were used to improve PIV signals. Basically, the seeding density and camera double-exposure sampling interval time constrain the spatial resolution of the PIV measurements. Seeding has to be added repeatedly to the glass enclosure to replenish particle density for PIV measurements. For the near field, across-the-slot flow database, a total of 72 phases of flow measurement were collected in 5 degree increments. In along-the-slot measurements, PIV samples at 18 phases were collected in 20-degree phase increments. For each PIV measurement, 400 samples are taken to estimate phase averaged statistics. Convergence reaches steady state between $100 \sim 200$ samples as shown in (Figure 6) 


\section{Results}

Flow field measurements are obtained after 30 minutes of actuator warm-up to reach steadiness. The temperature inside the cavity increases $12 \sim 13{ }^{\circ} \mathrm{F}$ above the ambient air and remains steady. The displacement waveforms from the diaphragm, follow a sine function, and are delayed about $50 \sim 60$ degrees behind the driver signal as shown in (Figure 7). Pressure waveforms inside the cavity contain higher harmonics. This harmonic component may be generated through nonlinear processes in the interior of cavity geometry and their presence appears over a range of diaphragm inputs. Both displacement and jet exit velocity signals are governed by simple oscillatory waveforms. Therefore the presence of the harmonics may be of secondary importance to the strength of synthetic jet produced.

The peak pressure in the cavity varies almost linearly with the diaphragm peak-to-peak displacement. In fact within our test range, Figure 8 shows a closer linear fit between the square root of the peak pressure and the peak-topeak displacement. Since the jet mass flux is a function of the diaphragm displacement amplitude and the oscillating frequency, the jet velocity emanating from the slot, and the static pressure in the cavity should be proportional to the diaphragm amplitude. We will show later that, in limited cases, the jet velocity properties may be scaled by either the peak-to-peak displacement or the square root of the peak pressure.

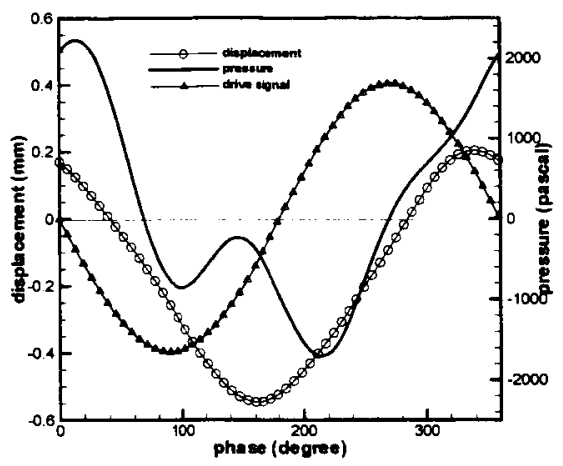

Figure 7. Waveforms of drive signal, diaphragm displacement, and cavity pressure.

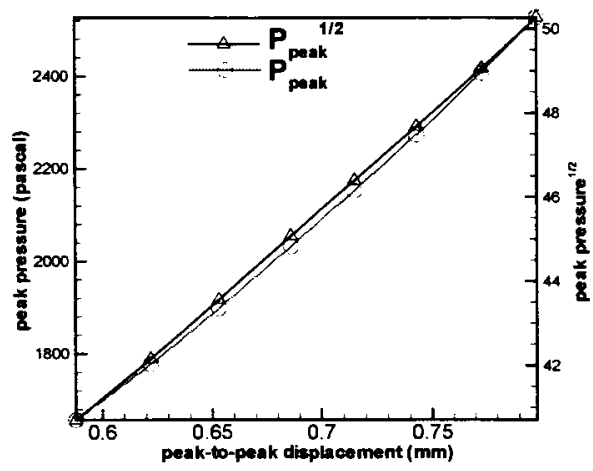

Figure 8. Cavity pressure as function of diaphragm displacement.

\section{A. Synthetic Jet Flow Field}

The discussion of the synthetic jet flow field in this section is based mostly on the extensive flow database from the PIV measurements. By design, PIV collects the spatial flow field information. Profiles of the jet velocity at the center of the slot from hot-wire and LDV measurements will be compared with PIV. We will discuss the flow field in a plane across the slot at $x=0$ to show the 2D properties of the jet, and the flow field in a plane along the slot at $y$ $=0$ to show the 3D structures of the plane jet with a finite span.

PIV measurements covered three configurations including planes with field-of-view: (1).across the slot at high spatial resolution (for near field data), (2) across the slot at lower spatial resolution (far field data), and (3) along the slot at low spatial resolution. The plane jet flow consists of a pulse train of high speed fluid projected from the rectangular slot accompanied by a pair of vortex rows that develop about half slot width above the slot on both sides of the exit. An elongated mean plane jet emerges when the flow fields are time or ensemble averaged over the driving cycle. Figure 9(a) shows the mean jet (streamwise) velocity contours at $x=0$. Near the exit, the jet is a narrow with nearly vertical motion. The jet turns into a wider, more diffusive jet about $12 h$ downstream, as shown in Figure 9(b). The two stage development of synthetic jets in the streamwise direction have been observed in earlier studies (Bera, et. al. $)^{11}$. In the plane at $y=0$, a more complicated plane jet structure is developed, as shown in Figure 9(c). Again near the jet exit, the flow emanates in a uniform, straight-up fashion. Then at about $4 h$ height, the end vortex starts rolling toward the center of the jet. The plane jet starts developing three dimensional structures. The slant rolling of the end vortex may be connected to the wide jet spreading downstream in the other plane. For CFD validation using $2 \mathrm{D}$ modeling, the current database is therefore limited to the region within $8 h$ distance from the jet exit for flow field comparison. In 3D modeling, however, the challenges should include the three dimensional development of the entire vortex ring around the plane jet. The symmetry of the flow field about the mid-point of the slot in x-z plane is clearly seen in Figure 9(c). The current database is limited to a single flow regime of a synthetic jet operating under a fixed input. 
In comparison between Figure 9(a) and Figure 9(b), obviously the high speed jet core at the near field is not resolved properly by the low spatial resolution PIV setting which behaves like a low pass filter to smear the velocity gradient at the jet core.

(a)

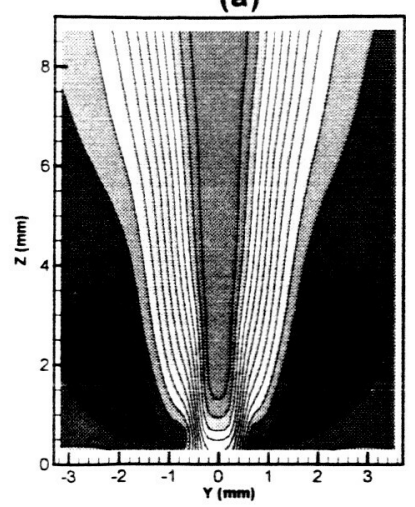

(b)

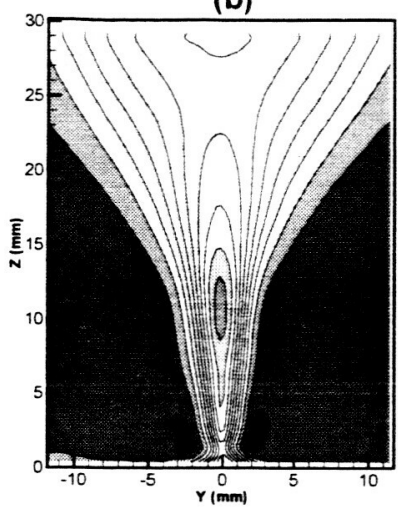

(c)

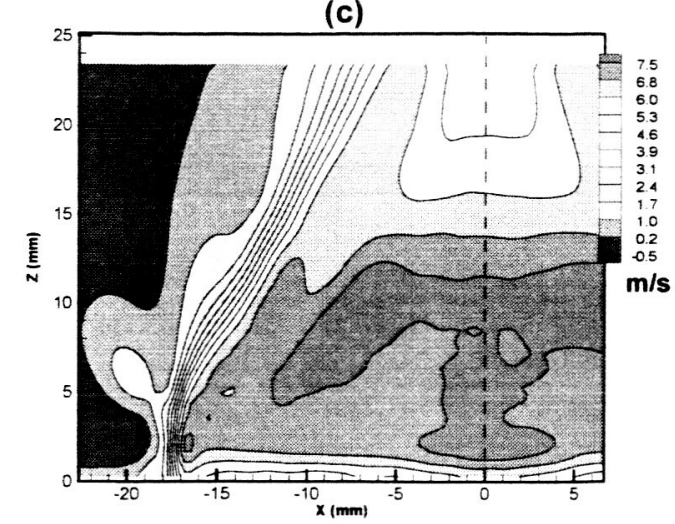

Figure 9. Mean jet contours. (a) across the slot at $x=0$, close-up view, (b) across the slot at $x=0$, large field of view, (c) along the slot at $y=0$, large field of view.

The phase-averaged flow field presents a statistically smoothed motion of the synthetic jet over the whole cycle. There are 72 phases=averaged means collected from this database at high resolution, and 18 phases at low resolution. Figure 11 presents four samples of phase-averaged synthetic jet flow fields from three fields of view.. In the first example, the jet is beginning to emerge from the slot (top figures) at the phase of $10^{\circ}$ referenced to the input sine function. The remains of the prior jet pulse at far field can still be seen in view (b) and (c). At a phase of $100^{\circ}$, there is a pair of vortices that move with the jet downstream. This vortex pair is a cross-section of the long and narrow vortex ring around the plane jet. In the third sample at of phase at $180^{\circ}$, the suction cycle takes place near the slot while the jet fluid is still moving downstream in far field. Finally, at a phase of $280^{\circ}$, the suction action extends upward but is terminated near $\sim 4 h(\sim 4-5 \mathrm{~mm})$ above the slot. From the side view (c), the plane jets show the growth of the edge vortex with height., moving toward the center along with the jet fronts, which are slightly buckled at the center.

Within each zone of the two jet expansion stages shown in Figure 9(b), the jet fluid is convected downstream at different speeds. From the trajectories of the height of the jet peak velocity and at various phases throughout the cycle (Figure 10), the jet convection speed is estimated to be about $14 \mathrm{~m} / \mathrm{s}$ in the near field (first stage), and then reduced to $\sim 4 \mathrm{~m} / \mathrm{s}$ in the far field (second stage). The vortex pair is moving at the same convection speed as the jet. In the first stage, the convection speed or velocity is greater than the jet mean velocity ( $\sim 8$ $\mathrm{m} / \mathrm{s})$, but less than the jet maximum velocity $(\sim 40 \mathrm{~m} / \mathrm{s})$. As the jet expands and weakens in the second stage, all three velocities start to converge. The implication is that the jet flow increases from the induced motion of the vortex pair in the near field. Downstream, both the jet and the vortex pair are weakened by turbulent diffusion. Therefore, the jet mean velocity and the convection speed are nearly

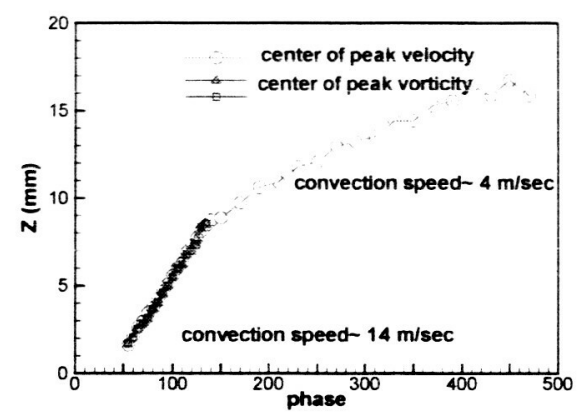

Figure 10. Trajectories of jet and vortex. equal. Finally, the jet reduces in strength and reaches its limit at about $12 \sim 13 h(\sim 15-16 \mathrm{~mm})$ from the exit. Beyond that point, the jet slug is completely diffused into a nearly steady motion. Smith and Swift ${ }^{14}$ conjectured the difference in the trajectories in the two stages, hence the convection speeds, coming from the turbulent transition of the vortex pair.

Besides the end effects at the edge of the exit, the 2D plane jet is expected to evolve into $3 \mathrm{D}$ structures through instabilities. Smith and Glezer ${ }^{9}$ recorded, by flow visualization, secondary vortical structures in the plane of the jet along the slot with an average spacing about $2.5 \mathrm{~h}$. From instantaneous flow samples by PIV, these finer structures develop when the jet moves downstream. Near the exit, the jet flow is relatively smooth and uniform, and the jet front develops wavy profiles downstream, as shown in Figure 12. 
(a)

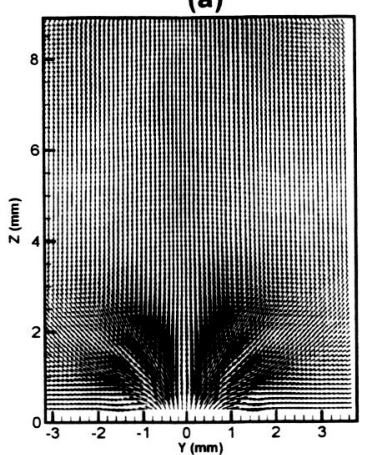

(a)

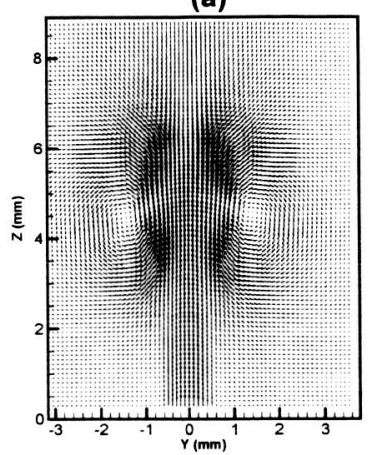

(a)

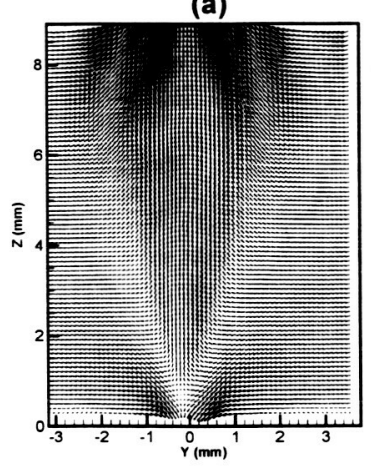

(a)

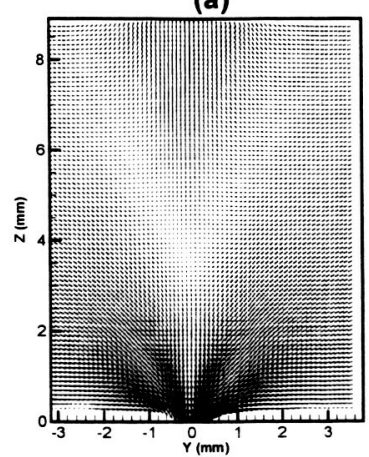

(b)

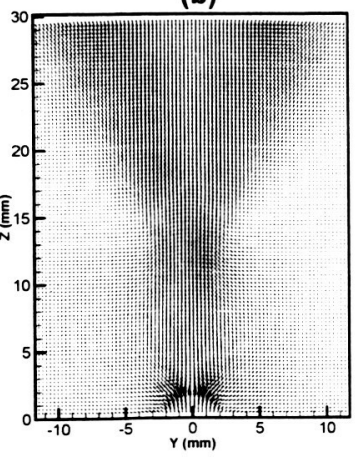

(b)

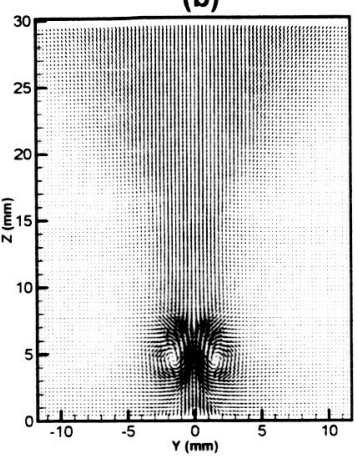

(b)

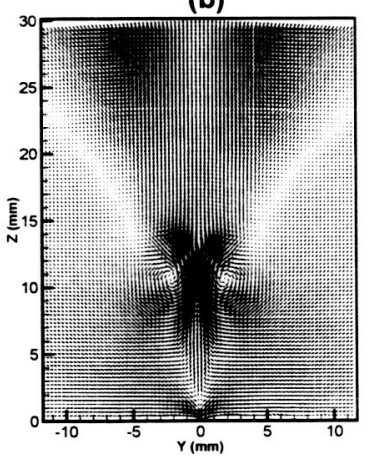

(b)

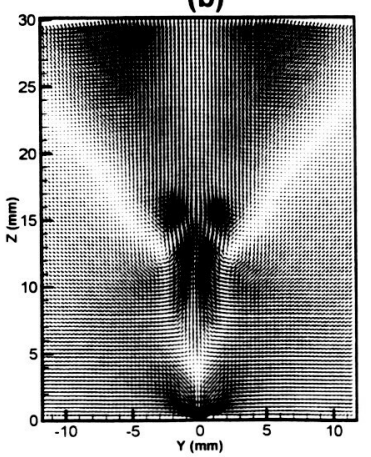

(c)

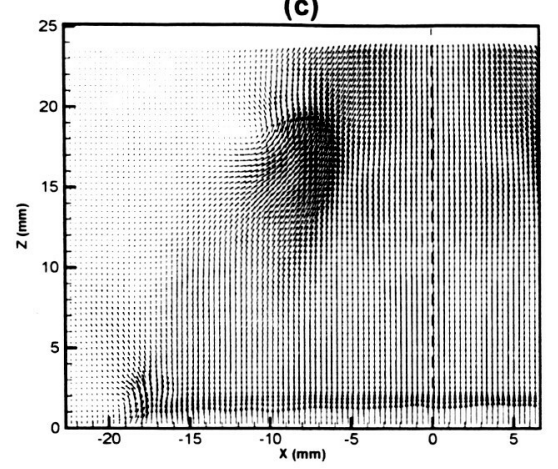

(c)

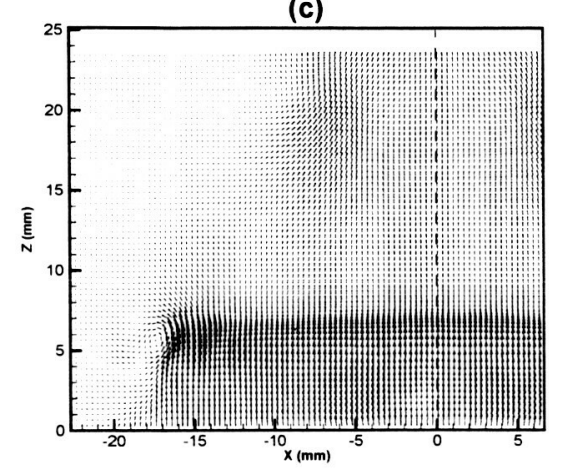

(c)

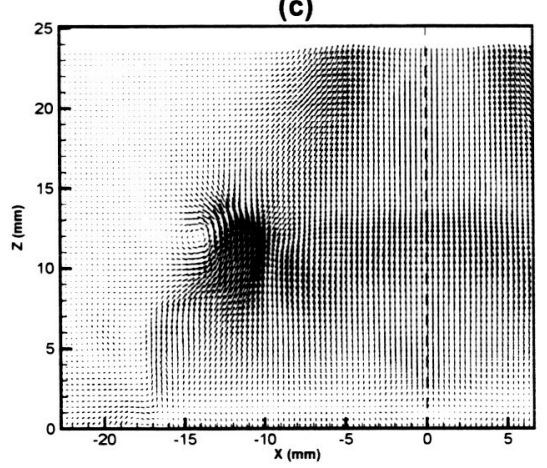

(c)

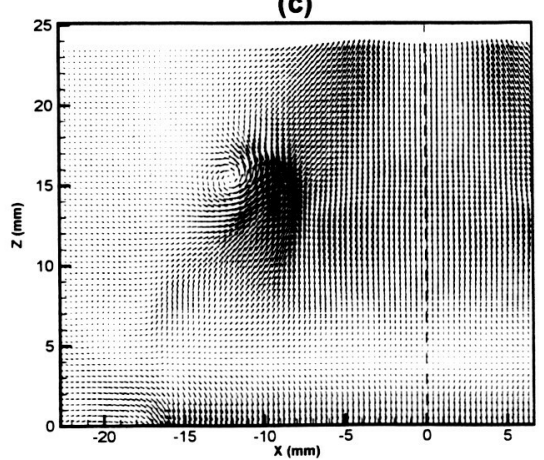

Figure 11. Phase averaged vector field: (a) close-up view at $x=0$ plane, (b) large field-of-view at $x=0$ plane, (c) large field of view along the slot at $y=0$ plane. Top row, phase $=10^{\circ}$. Second row, phase $=100^{\circ}$. Third row, phase $=180^{\circ}$. Bottom row, phase $=280^{\circ}$. 
(a)

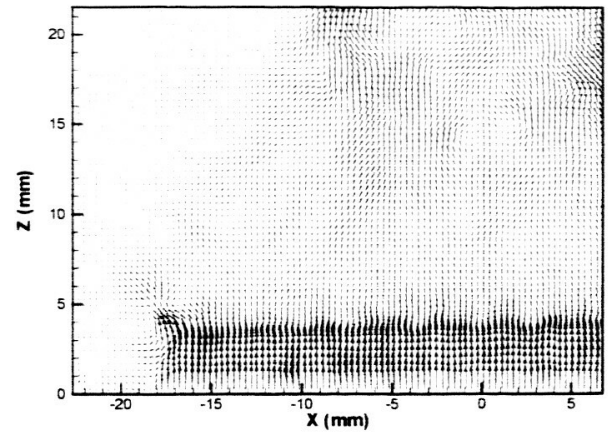

(b)

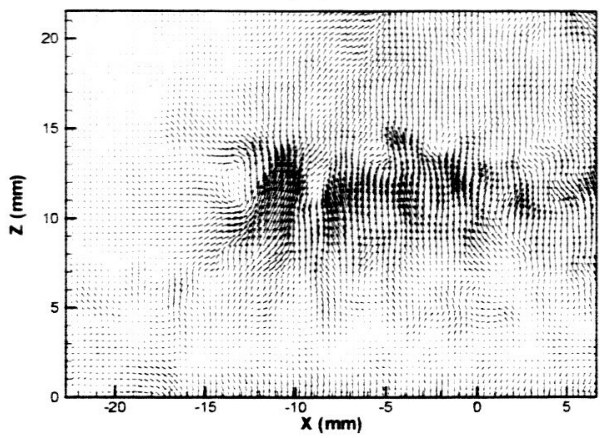

Figure 12. Instantaneous flow field along the jet slot sampled at: (a) phase $=120^{\circ}$, (b) phase $=180^{\circ}$.

\section{B. Comparison between Hot-wire, LDV and PIV}

The mean jet velocity profiles from all three measurement techniques at the center axis of the slot are shown in Figure 13. LDV and PIV profiles show a similar distribution. Because the LDV data were taken at a higher displacement amplitude, the LDV data needs to be rescaled to properly match the PIV profile through displacement scaling. The hot-wire data show lower means near the exit, then agree with LDV and scaled PIV data farther away from the slot exit. The zigzag of the hot-wire profile takes place from signal derectification due to the flow reversal within $4 h$ distance from the jet exit. During the workshop it was suggested that this was due to a temperature influences and could be corrected. Even with the temperature compensation, the correction is still not sufficient to account for the over-estimation.

The comparison profiles of jet maximum velocities, between hot-wire measurements, LDV, and PIV is similar to the mean profiles. Maximum velocity refers to the peak velocity within the driving cycle at each measurement station along the jet axis. The agreement in profile shape between LDV and PIV is good except the magnitudes are off. When the LDV profile is rescaled by the displacement amplitudes, the two profiles collapse. Scaling the maximum velocity using $p(\text { peak })^{1 / 2}$ is found to be equally effective. LDV and PIV measurements show that the maximum velocity in the jet increase with height to about $z \sim 4 h(\sim 5 \mathrm{~mm})$. Hot-wire profile shows higher maximum near the exit. It peaks near $z \sim 1.5 h(\sim 2 \mathrm{~mm})$ then decays fast. Downstream of $z>15 h(\sim 19 \mathrm{~mm})$, the agreement between the mean profiles from hot-wire and LDV data matched well. Interestingly, the maximum velocity increases slightly at $z \sim 28 h(36 \mathrm{~mm})$. This minor reversal is also seen in the PIV mean in Figure 9.

Another example of scaling using displacement amplitude is shown in Figure 15. The velocity waveform from LDV and PIV matches at $z=2$ and $5 \mathrm{~mm}$ on the centerline. However, we also found, in a separate test, that this scaling fails to apply to jets at a lower strength. Comparison of maximum velocity profiles from three actuator input forcing levels shows the two stronger jet profiles are correctly scaled, but the third and weaker jet failed to match their amplitudes (Figure 16). Nevertheless, the agreement improves near the jet exit.

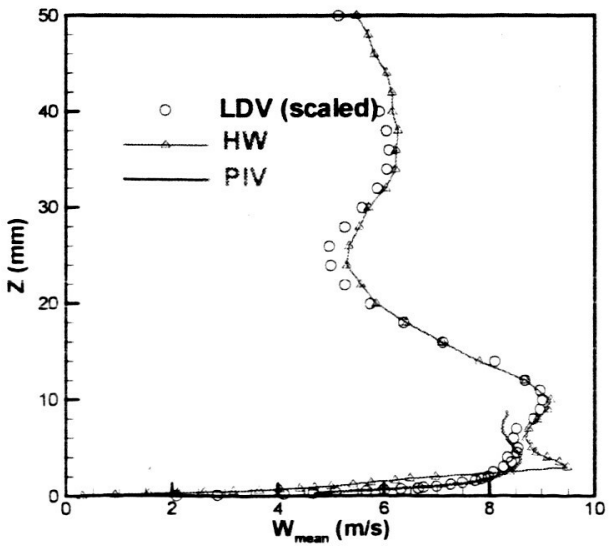

Figure 13. Mean jet velocity profiles, averaged over one cycle.

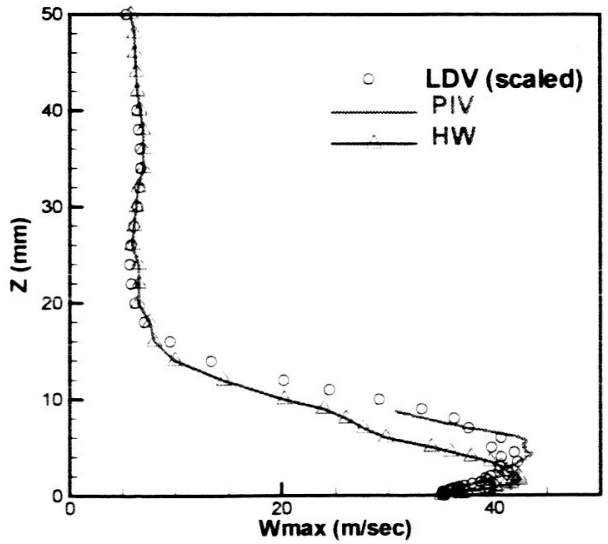

Figure 14. Maximum jet velocity profiles.

American Institute of Aeronautics and Astronautics 
In an earlier observation of the jet trajectories, the vortex pair motion apparently induces higher peak velocity within the jet. Jet velocity at the exit, on the other hand, scales straightforwardly with the diaphragm displacement at a fixed drive frequency. The vortex strength thus may not be scaled properly by just diaphragm displacement alone. Therefore the ability to scale the velocity profiles could be very limited. However, through this scaling, the comparison between LDV and PIV data moves much closer.

(a)

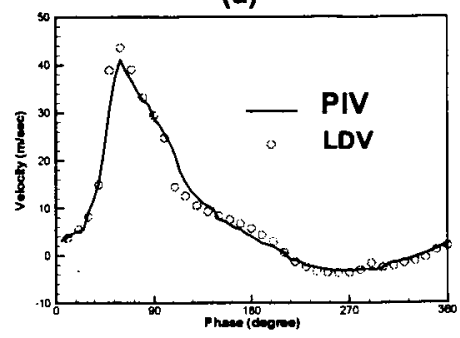

(b)

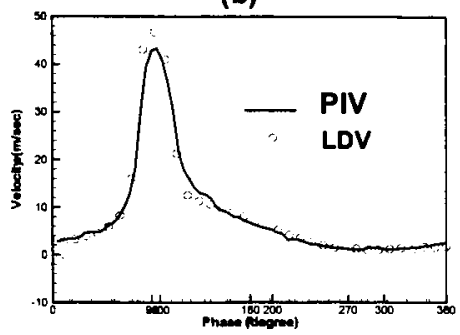

Figure 15. Jet velocity waveform at: (a) $z=2 \mathrm{~mm}$, and (b) $\mathrm{z}=5 \mathrm{~mm}$. LDV data scaled by the displacement.

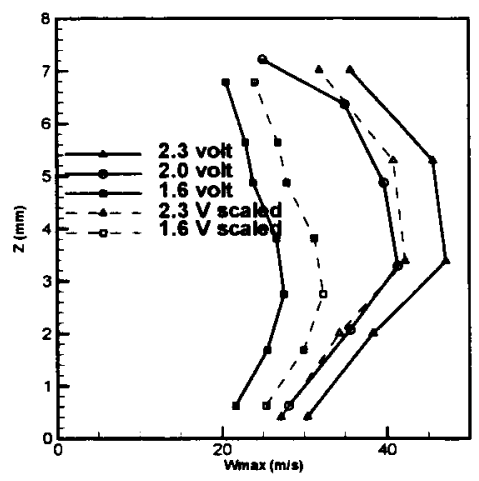

Figure 16. Scaling of maximum velocity.

\section{Conclusion}

The present study provides a detailed flow field database of a synthetic jet flow in quiescent air for the CFD validation study. PIV measurements provide extensive phase-averages of the flow field at planes normal and parallel to the jet slot, supplemented by actuator pressure, temperature, and diaphragm displacement measurements for boundary conditions. In addition, local velocity profiles by hot-wire and LDV measurements provide a crosscheck of PIV performance.

The isolated plane jet starts with a two-dimensional uniform motion in the near field, accompanied by vortex pairs parallel to the slot and at the edge.. The vortex rings at the edge start to roll toward the center of the plane downstream, and the jet flow turns to three dimensional. Views of the flow field along the slot show highly fluctuating flows as jets advancing downstream. In the cross-the-jet view, the jet expands downstream in two stages, giving evidence of different flow process in each stage and possible transition to turbulence, which was speculated in previous synthetic jet flow studies. A 2D modeling may only be sufficient or valid within the first stage of the jet development. The suction action of the jet is stopped at short distance from exit. A weak, broad, nearly continuous, jet motion prevails in the far field. Instantaneous flow field measurements show strong unsteadiness as jets emerged from the slot. Phase-averaged flow field measurements statistically converge to mean jet motions.

In the near field, close agreements are seen between the LDV and PIV when jet velocity profiles are scaled by the peak cavity pressure or diaphragm displacement. However, this scaling is not universal. Hot-wire measurements are off in comparison with LDV and PIV near the exit, but the comparison improved downstream, where the jet oscillatory amplitudes are reduced significantly. 
The PIV technique is proven to be effective in collecting an extensive flow field database. which may cover a wide ranges of field of views of interest. It also reduces the testing time and costs to a manageable level. Although, statistics estimated from PIV measurements matches flow data from hot-wire and LDV in this study, in general, the limit of PIV is embedded in its reliance on the performance of seeding particles, optical imaging, and pixel resolution. For applications of CFD validation, we have demonstrated the capability of PIV to obtain reliable quantitative information on flow structures and processes.

\section{References}

'Langley Research Center Workshop on "CFD Validation of Synthetic Jets and Turbulent Separation Control," URL: http://cfdval2004.larc.nasa.gov

${ }^{2}$ Seifert, A. and Pack, L. G., "Active Control of Separated Flows on Generic Configurations at High Reynolds Number," AlAA Paper 99-3403, 1999.

${ }^{3}$ Ho, C.-M., and Tai, Y.-C., "Review: MEMS and Its Applications for Flow Control," Journal of Fluids Engineering, Vol. 118, No. 3, pp. 437-447, 1996.

${ }^{4}$ Jacobson. S. A., and Reynolds, W. C., "An Experimental Investigation Toward the Active Control of Turbulent Boundary Layers," Thermosciences Division, Rept. TF-64< Dept. of Mechanical Engineering, Stanford Univ., Stanford, CA. 1995.

${ }^{5}$ Lin, Y.-L., Chyu, M. K., and Shih, T. I-P, "Skin-Friction Reduction Through Micro Blowing," AIAA Paper 98-0359, 1998.

${ }^{6}$ Smith, B. L., and Glezer, A., "The Formation and Evolution of Synthetic Jets," Physics of Fluids, Vol. 10, No. 9, pp.2281$2297,1998$.

${ }^{7}$ Crook, A., Sadri, A. M., and Wood. N. J., "The Development and Implementation of Synthetic Jets for the Control of Separated Flow," AIAA Paper 99-3176, 1999.

${ }^{8}$ Mallinson, G., Hong. G., and Reizes, J. A., “Some Characteristics of Synthetic Jets," AlAA Paper 99-33673, 1999.

${ }^{9}$ Chen, F.-J., Yao, C., Beeler, G. B., Bryant. R. G., and Fox, R. L., "Development of Synthetic Jet Actuators for Active Flow Control at NASA Langley," AIAA Paper 2000-2405, 2000.

${ }^{10}$ Muller, M. O., Bernal, L. P., Miska, P. K., Washabaugh, P. D., Chou, T., Parviz, B., Zhang, C., and Najafi, K., "Flow Structure and Performance of Axisymmetric Synthetic Jets," AIAA Paper 2001-1008, 2001

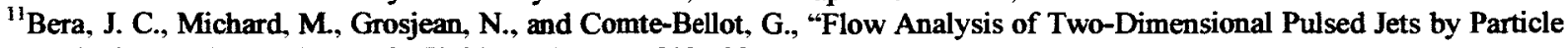
image Velocimetry," Experiments in Fluids, Vol.31, pp 519-532, 2001.

${ }^{12}$ Guy, Y., McLaughlin, and Morrow, J. A., "Velocity Measurements in a Synthetic Jet". AIAA Paper 2001-0118, 2001.

${ }^{13}$ Guy, Y., McLaughlin, and Albertson, J. A., "Effect of Geometric Parameters on the Velocity Output of a Synthetic Jet Actuator," AIAA Paper 2002-0126, 2002.

${ }^{14}$ Smith, B. L., and Swift, G. W., "Synthetic Jets at Large Reynolds Number and Comparison to Continuous Jets," AIAA Paper 2001-3030, 2001, 